\title{
Venoarterio-Venous (VA-V) ECMO Configuration: A Single-Center Experience
}

\author{
Casey Kukielski ${ }^{1}$, Carlton Davis ${ }^{2}$, Asif Saberi ${ }^{3}$, and Sanjay Chaudhary ${ }^{4}$ \\ ${ }^{1}$ University of Maryland Medical Center \\ ${ }^{2}$ Emory University \\ ${ }^{3}$ WellStar Health System \\ ${ }^{4}$ Mayo Clinic Hospital Jacksonville
}

September 26, 2021

\begin{abstract}
Patients in respiratory failure on VV ECMO may develop cardiovascular dysfunction necessitating additional hemodynamic support, while patients in cardiovascular failure on VA ECMO may require additional respiratory support for concurrent gas exchange abnormalities. A hybrid venoarterio-venous (VA-V) configuration provides both cardiac support via a traditional arterial reinfusion cannula and respiratory support via an additional venous reinfusion limb. We describe our single center experience using VA-V ECMO for patients ( $\mathrm{n}=14$, median age 54) with combined cardiopulmonary failure or differential hypoxemia. Patients were treated with ECMO support for a median of 148.2 (IQR 122.6 - 174.4) hours, consisting of 0 (IQR $0-1.8$ ) hours of VA and 92.4 (IQR $58-115$ ) hours of VA-V followed by 46 (IQR $0-95.5$ ) hours of VV support. Of these 14 patients, 11 survived to decannulation $(79 \%)$ and 9 survived to hospital discharge (64\%).
\end{abstract}

\section{TITLE PAGE}

Venoarterio-Venous (VA-V) ECMO Configuration: A Single-Center Experience

Authors

Casey Kukielski MD Graduate Medical Education, Anesthesiology Residency Program, University of Maryland School of Medicine, Baltimore, Maryland

C. Jarrett Davis MD Graduate Medical Education, Anesthesiology Residency Program, Emory University Hospital, Atlanta, Georgia

Asif Saberi MD Division of Critical Care Medicine, Wellstar Kennestone Hospital, Marietta, Georgia.

Sanjay Chaudhary MBBS MD (Corresponding Author) Department of Critical Care Medicine, Mayo Clinic, Jacksonville, Florida

Chaudhary.sanjay@mayo.edu https://orcid.org/0000-0003-4127-5572

Funding

None

Conflict of interest

None

IRB approval 
Wellstar Research Institute Institutional Review Board (1624214-1)

Informed consent

Not applicable

Clinical trial registration

Not applicable

\begin{abstract}
Patients in respiratory failure on VV ECMO may develop cardiovascular dysfunction necessitating additional hemodynamic support, while patients in cardiovascular failure on VA ECMO may require additional respiratory support for concurrent gas exchange abnormalities. A hybrid venoarterio-venous (VA-V) configuration provides both cardiac support via a traditional arterial reinfusion cannula and respiratory support via an additional venous reinfusion limb. We describe our single center experience using VA-V ECMO for patients $(\mathrm{n}=14$, median age 54) with combined cardiopulmonary failure or differential hypoxemia. Patients were treated with ECMO support for a median of 148.2 (IQR 122.6 - 174.4) hours, consisting of 0 (IQR 0 - 1.8) hours of VA and 92.4 (IQR $58-115$ ) hours of VA-V followed by 46 (IQR $0-95.5$ ) hours of VV support. Of these 14 patients, 11 survived to decannulation (79\%) and 9 survived to hospital discharge $(64 \%)$.
\end{abstract}

\title{
Keywords
}

Extracorporeal membrane oxygenation; ECMO; VA-V; VV; VA; hybrid; venoarterio-venous

\section{Introduction}

Extracorporeal membrane oxygenation (ECMO) is a form of temporary cardiopulmonary support used in severe cardiovascular or respiratory failure. Using large cannulae placed in major blood vessels, deoxygenated venous blood is circulated through an extracorporeal circuit, which includes an oxygenator that conducts efficient gas exchange. This circulation is supported by pressure generated by a pump. The oxygen-rich blood is then reinfused back into the body via another large blood vessel.

Veno-venous (VV) ECMO is used primarily for respiratory support whereby oxygen-rich blood is reinfused into a large vein. Veno-arterial (VA) ECMO provides hemodynamic support by reinfusing this oxygen rich blood into the arterial circulation through a large cannula usually placed in a major distributary of the aorta. It is not uncommon for patients who primarily have respiratory failure to also have significant cardiovascular compromise. The latter usually improves once adequate gas exchange is restored on VV ECMO. However, not infrequently there is concomitant and significant cardiovascular and respiratory failure. For example, patients with severe respiratory failure on VV ECMO may develop cardiomyopathy requiring significant cardiovascular support. Conversely, patients with primarily cardiovascular failure on VA ECMO may have or develop concomitant severe respiratory failure due to pulmonary edema from elevated left atrial pressure or a primary injury to the lungs. In the absence of significant cardiovascular recovery, oxygenation of the proximal aorta and its distributaries is largely from the oxygen rich blood reinfused by the ECMO circuit. In patients on VA ECMO, recovery of heart function with persistent severe lung injury leads to "differential hypoxemia" where ejection of poorly oxygenated blood from the left ventricle competes with retrograde oxygenated blood flow from the ECMO circuit, leading to myocardial and cerebral ischemia.(1-8) This is detected by comparing oxygenation from a right radial arterial sample with one obtained from the left. As LV function recovers, the right sided arterial blood sample will have relatively lower oxygen content reflecting native lung dysfunction. With further recovery, the "mixing point" of blood may move further down the aortic arch with lower oxygen content of both compared to the femoral arteries.

To address these perfusion requirements, clinicians have developed "hybrid" configurations by placing additional cannulae.(9) The most common of these is venoarterio-venous (VA-V) ECMO, which consists of a single venous drainage cannula and both arterial and venous reinfusion limbs. In patients with secondary 
heart failure (on VV ECMO), a femoral, subclavian, or axillary artery cannula is added to provide adequate circulatory support. In differential hypoxemia (on VA ECMO) an internal jugular or subclavian vein catheter is added to deliver oxygenated blood to the pulmonary circulation.(4) Using an adjustable clamp on the venous reinfusion limb, arterial and venous reinfusion flows are titrated until adequate hemodynamics and oxygenation are obtained. $(10,11)$

The number of patients being treated with ECMO has dramatically increased over the last decade, and its use in our ICU has followed a similar trend. $(4,12)$ While a few case series have provided initial descriptions of patients requiring VA-V ECMO, there remains a paucity of literature on the subject. $(10,11,13,14)$ We report our hospital's experience with VA-V ECMO to further characterize the patient population, indications, and outcomes associated with this hybrid configuration.

\section{Methods}

We retrospectively reviewed all adult patients placed on VA-V ECMO, for any part of the run, at our institution from June 1, 2016 to December 10, 2019. The decision to place patients on ECMO and choice of configuration was made by our institution's ECMO team which consists of intensivists with special training and proficiency in ECMO. Through a review of the electronic medical records, data describing demographic features, comorbidities, and ECMO-specific information were reviewed systematically. Continuous variables were reported as median with interquartile range and categorical variables were summarized by frequency of observation. This study was approved by the Wellstar Research Institute Institutional Review Board (1624214-1).

\section{Results}

During the study period, there were 181 patients treated with ECMO, of which, 72 had cardiovascular failure and were treated with VA ECMO. Of these, 14 patients were placed on VA-V ECMO. At our institution, all patients on VA ECMO receive a right radial, brachial or axillary artery catheter for blood gas sampling (except for patients who have a right axillary side-graft for reinfusion). There were more males (11/14, 79\%) than females in this cohort. Their median age was 54 (range $18-75$ ) with median BMI of 30.3 (range $25-$ 54). Patient characteristics are summarized in Table 1 .

Underlying diseases included myocardial infarction (4/14), cardiac arrest (3/14, two with concurrently diagnosed pneumonia [PNA]), pulmonary embolism (2/14), anti-hypertensive overdose $(2 / 14$, one with coexisting pneumonia), decompensated pulmonary arterial hypertension [PAH], penetrating chest injury, and postpericardiotomy syndrome. Seven patients (50\%) were transferred to our institution for ECMO, while 50\% (7/14) of patients suffered pre-ECMO cardiac arrest. Our study population had a median SOFA-0 score of 15 (predicted ICU mortality of $95.2 \%$ ) and the last arterial blood gas prior to ECMO cannulation had a median $\mathrm{pH}$ of $7.20, \mathrm{PaO}_{2} / \mathrm{FiO}_{2}$ ratio of 73 , and $\mathrm{PaCO}_{2}$ of 50 ; one patient did not have a documented ABG prior to cannulation. Five patients had a left ventricular ejection fraction [?] 30\% on pre-ECMO echocardiogram, and five patients had moderately or severely reduced right ventricular ejection fraction (RVEF); two patients did not have a documented pre-ECMO transthoracic echocardiogram (TTE), and the RVEF of two patients was not assessed due to technical limitations.

Underlying disease, initial ECMO indication, and indication for VA-V conversion are displayed in Table 2 . Seven $(50 \%)$ patients were initiated on VA ECMO with a median SAVE score of -12 (risk class V, predicted in-hospital survival of 18\%), and the remaining seven patients were placed directly on VA-V ECMO due to present or imminent combined cardiopulmonary failure. The majority of patients initially cannulated on VA ECMO were quickly transitioned to a VA-V configuration, however three patients were on VA ECMO for a significant amount of time. None of the patients in our study population were initiated on a veno-venous configuration. Sequence of ECMO configurations with cannula size and locations are shown in Table 3 . Two patients were cannulated at outside hospitals prior to transfer, and the cannula sizes for one of these patients were not documented in our EMR. A superficial femoral artery distal perfusion cannula was placed in 9 of $13(69 \%)$ patients who were cannulated via the femoral artery, and four patients (29\%) had an Impella in place while on ECMO. 
Arterial blood gas data is shown in Table 4. On Day 1 of ECMO, our patients had a median $\mathrm{PaO}_{2}$ of 271 on a median ECMO flow of $4.22 \mathrm{~L} / \mathrm{min}$. Indications for VA to VA-V conversion included differential hypoxemia $(5 / 7,71 \%)$, persistent hypoxemia, and respiratory failure secondary to diffuse alveolar hemorrhage.

ECMO adjuvants, anticoagulation strategy, and blood product administration are shown in Table $\mathbf{5}$. One patient was only anticoagulated with Impella purge solution, eight $(57 \%)$ received a heparin infusion, and five $(36 \%)$ were placed on Argatroban infusion (two of these patients were transitioned from heparin due to concern for HIT). Anticoagulation was held due to bleeding in $79 \%(11 / 14)$ of patients at some point in their ECMO run, and a median of 7.5 units of PRBCs and 2.5 units of platelets were transfused while on ECMO.

Of the 11 patients that demonstrated cardiac and pulmonary recovery allowing for weaning of ECMO support, eight (73\%) were transitioned to VV prior to decannulation and three $(27 \%)$ were decannulated directly from VA-V. The first arterial blood gas following ECMO had a median $\mathrm{pH}$ of 7.39, $\mathrm{PaO} 2 / \mathrm{FiO} 2$ ratio of 262, and $\mathrm{PaCO} 2$ of 49. Six of eleven patients had documented post-ECMO transthoracic echocardiograms, which are shown in Table 6 .

Complications are listed by subcategory in Table 7 . In order of frequency, these included acute kidney injury $(93 \%)$, clinically significant bleeding (50\%), thrombotic non-circuit (36\%), limb ischemia (36\%), infection (29\%), neurologic (29\%), and mechanical/circuit (14\%). Two patients with limb ischemia required intervention: one patient required bilateral below knee amputation and another with SFA occlusion required thrombectomy. All 4 infectious complications were due to pneumonia. With regards to neurologic complications, 2 patients had embolic strokes and 2 patients developed anoxic brain injury. Additionally, mechanical/circuit complications occurred in 2 patients: 1 patient had oxygenator fibrin accumulation requiring circuit exchange, and another had distal perfusion cannula clotting that required catheter exchange.

Patient outcomes are listed in Table 8 . VA-V support was utilized for 92.4 out of 148.2 total ECMO hours (median values with first and third quartiles displayed). Causes of death on ECMO included cor pulmonale, asystolic cardiac arrest, and withdrawal of care due to persistent cardiogenic shock as well as ARDS with severe hypoxic respiratory failure. Two patients did not survive from decannulation to discharge due to respiratory failure and withdrawal of care in the setting of anoxic brain injury. A total of $79 \%(11 / 14)$ of patients survived to ECMO decannulation and 64\% (9/14) survived to hospital discharge, with $82 \%$ of decannulated patients surviving to discharge. One patient was transferred back to their original hospital and the remaining patients were discharged to an extended care facility (11\%), physical rehabilitation facilities $(33 \%)$, or home $(44 \%)$. Cumulatively, these patients spent a median of 12.5 days on a ventilator, 14.5 days in the ICU, and 22 days in the hospital with a median of 12 ventilator free, 28.5 ICU free, and 1.5 hospital free days in 60 .

\section{Discussion}

The dramatic increase in ECMO utilization over the last decade has provided a wealth of data and experience that has enhanced physician comfort with treating these complicated patients. However, it has also brought unique challenges associated with increasingly complex physiology. Some patients supported with VA ECMO may develop differential hypoxemia resulting in poor cerebral, myocardial, and upper body oxygenation and many whose initial presentation is combined cardiopulmonary failure cannot be adequately supported with standard percutaneous configurations. While central cannulation is another possible strategy, it is usually not a preferred approach unless the patient is already undergoing cardiac surgery or has severely diseased peripheral arteries precluding placement of adequately sized cannulae for optimal support. Another strategy used in such circumstances is axillary artery cannulation, which can be a good alternative to femoral artery reinfusion. Its advantages include less differential hypoxemia, less secondary left ventricular distension and greater mobility on ECMO. However, it is usually performed utilizing a side graft technique, is not suitable for urgent or emergent situations, and can potentially lead to upper extremity hyperperfusion and compartment syndrome. In a patient supported with percutaneous VA ECMO utilizing a femoral arterial reinfusion cannula, an additional venous reinfusion cannula ensures oxygenated cardiac preload and hemodynamic support that satisfies these physiologic requirements. 
As with previous studies, our cohort is small and heterogeneous although largely older and obese with frequent cardiac and pulmonary comorbidities. The median SOFA-0 score of 15 and SAVE score of -12 (95.2\% predicted ICU mortality and $18 \%$ in-hospital survival respectively) highlight the extreme severity of illness prior to ECMO cannulation.(15) Half of our patients were placed directly on VA-V due to comorbid cardiopulmonary failure at presentation, and the remaining patients were initiated on VA with differential hypoxemia as the most common indication for VA to VA-V conversion. Although it was initially hypothesized that VA-V conversion may reduce neurologic complications, the $14 \%$ incidence of anoxic brain injury is consistent with other reports across both standard and VA-V configurations. ${ }^{11}$ However, the survival rate of $64 \%$ compares favorably with 2016 ELSO Report adult statistics (43-65\% in respiratory failure and 42-51\% in shock and cardiomyopathy) as well as previous VA-V case series (39-42.9\%). Notably, during the same period, three patients received an axillary graft for reinfusion as a substitute for traditional femoral arterial cannulation, but their data is not included in our analysis.

ECMO provides respiratory and circulatory support to facilitate native organ recovery (or less commonly bridge to transplant). As it is utilized in increasingly complex patients, it is not unexpected that the incidence of comorbid cardiopulmonary failure and sequelae such as differential hypoxemia will continue to grow. This is supported by the use of VA-V in 7.7\% (14 out of 181) of our ECMO patients from 2016 to 2019, compared to less than $1.5 \%$ in the largest previous case series. ${ }^{11}$ It is important to note that the cannulation strategy may not be fixed for the duration of ECMO support. Patient physiology, or clinical conditions and requirements, may change over time (e.g. the heart may recover faster than the lungs) and modifications in ECMO configurations may occasionally be necessary. The conversion from the initial ECMO strategy to a different modality should always be strongly considered if the patient's perfusion is inadequate, gas exchange is suboptimal, or complications result from the initial cannulation strategy. However, one should always be cautious with additional cannula placement due to the ongoing anticoagulation for ECMO, higher risk of bleeding (particularly in arterial vasculature), and risk of another port for infection or thrombosis.(9) Since the small number of patients with presentations prompting consideration of VA-V cannulation precludes larger randomized trials, clinical decision making is heavily influenced by smaller case series and expert opinion. Despite lack of reduction in neurologic complications, we believe that combined cardiopulmonary failure and differential hypoxemia necessitate additional venous reinfusion to supply oxygenated cardiac preload and subsequent upper body delivery. At minimum, our data indicates maintained survival with VA$\mathrm{V}$ cannulation, which is notable as these are the ECMO patients with the highest severity and complexity of illness. As such, we advocate for early consideration and proactive VA-V cannulation in these situations.

\section{Conclusion}

ECMO patients with recovering left ventricular function and persistent gas exchange abnormalities are at risk for developing differential hypoxemia. We describe an approach to identifying and treating differential hypoxemia and prophylactically utilizing VA-V configuration when the likelihood of differential hypoxemia is extremely high.

References

Uncategorized References

1. Abrams D, Combes A, Brodie D. Extracorporeal Membrane Oxygenation in Cardiopulmonary Disease in Adults. Journal of the American College of Cardiology. 2014;63(25, Part A):2769-78.

2. Bartlett RH, Roloff DW, Custer JR, Younger JG, Hirschl RB. Extracorporeal Life SupportThe University of Michigan Experience. JAMA. 2000;283(7):904-8.

3. Bisdas T, Beutel G, Warnecke G, Hoeper MM, Kuehn C, Haverich A, et al. Vascular Complications in Patients Undergoing Femoral Cannulation for Extracorporeal Membrane Oxygenation Support. The Annals of Thoracic Surgery. 2011;92(2):626-31.

4. Brasseur A, Scolletta S, Lorusso R, Taccone FS. Hybrid extracorporeal membrane oxygenation. Journal of Thoracic Disease. 2018;10 (Suppl 5):S707-S15. 
5. Brodie D, Bacchetta M. Extracorporeal Membrane Oxygenation for ARDS in Adults. New England Journal of Medicine. 2011;365(20):1905-14.

6. Combes A, Brodie D, Chen Y-S, Fan E, Henriques JPS, Hodgson C, et al. The ICM research agenda on extracorporeal life support. Intensive Care Medicine. 2017;43(9):1306-18.

7. Loftsgard TO, Newcome MD, Hanneman MR, Patch RK, 3rd, Seelhammer TG. Management of Neurogenic Pulmonary Edema and Differential Hypoxemia in an Adult Supported on Venoarterial Extracorporeal Membrane Oxygenation. J Cardiothorac Vasc Anesth. 2017;31(6):2170-4.

8. Mohite PN, Fatullayev J, Maunz O, Kaul S, Sabashnikov A, Weymann A, et al. Distal limb perfusion: Achilles' heel in peripheral venoarterial extracorporeal membrane oxygenation. Artif Organs. 2014;38(11):940-4.

9. Sorokin V, MacLaren G, Vidanapathirana PC, Delnoij T, Lorusso R. Choosing the appropriate configuration and cannulation strategies for extracorporeal membrane oxygenation: the potential dynamic process of organ support and importance of hybrid modes. European Journal of Heart Failure. 2017;19(S2):75-83.

10. Biscotti M, Lee A, Basner RC, Agerstrand C, Abrams D, Brodie D, et al. Hybrid Configurations via Percutaneous Access for Extracorporeal Membrane Oxygenation: A Single-Center Experience. ASAIO Journal. 2014;60(6):635-42.

11. Werner NL, Coughlin M, Cooley E, Haft JW, Hirschl RB, Bartlett RH, et al. The University of Michigan Experience with Veno-Venoarterial Hybrid Mode of Extracorporeal Membrane Oxygenation. ASAIO Journal. 2016;62(5):578-83.

12. Gerke AK, Tang F, Cavanaugh JE, Doerschug KC, Polgreen PM. Increased trend in extracorporeal membrane oxygenation use by adults in the United States since 2007. BMC Research Notes. 2015;8(1):686.

13. Stöhr F, Emmert MY, Lachat ML, Stocker R, Maggiorini M, Falk V, et al. Extracorporeal membrane oxygenation for acute respiratory distress syndrome: is the configuration mode an important predictor for the outcome? Interactive CardioVascular and Thoracic Surgery. 2011;12(5):676-80.

14. Ius F, Sommer W, Tudorache I, Avsar M, Siemeni T, Salman J, et al. Veno-veno-arterial extracorporeal membrane oxygenation for respiratory failure with severe haemodynamic impairment: technique and early outcomes. Interactive CardioVascular and Thoracic Surgery. 2015;20(6):761-7.

15. Chamogeorgakis T, Lima B, Shafii A, Nagpal D, Pokersnik J, Navia J, et al. Outcomes of axillary artery side graft cannulation for extracorporeal membrane oxygenation. Journal of Thoracic and Cardiovascular Surgery. 2013;145:1088-92.

\section{Hosted file}

Tables VA-V ECMO.docx available at https://authorea.com/users/437894/articles/539236venoarterio-venous-va-v-ecmo-configuration-a-single-center-experience 